will welcome expressions of interest from experts who wish to contribute.

The DECIDE-AI Steering Group

Baptiste Vasey ${ }^{1 凶}$, David A. Clifton ${ }^{2}$,

Gary S. Collins ${ }^{3,4}$, Alastair K. Denniston ${ }^{5}$, Livia Faes ${ }^{6,7}$, Bart F. Geerts ${ }^{8}$, Xiaoxuan Liu ${ }^{5,7}$, Lauren Morgan ${ }^{9}$, Peter Watkinson ${ }^{10}$ and Peter McCulloch ${ }^{1}$

${ }^{1}$ Nuffield Department of Surgical Sciences, University of Oxford, Oxford, UK. ${ }^{2}$ Institute of Biomedical Engineering, Department of Engineering Science, University of Oxford, Oxford, UK. ${ }^{3}$ Centre for Statistics in Medicine, Nuffield Department of Orthopaedics, Rheumatology and Musculoskeletal Sciences, University of Oxford, Oxford, UK. ${ }^{4} \mathrm{NIHR}$ Oxford Biomedical Research Centre, John Radcliffe
Hospital, Oxford, UK. ${ }^{5}$ University Hospitals

$\square$ Birmingham NHS Foundation Trust, Birmingham, UK. ${ }^{6}$ Eye Clinic, Cantonal Hospital Lucerne, Lucerne, Switzerland. ${ }^{7}$ Moorfields Eye Hospital NHS Foundation Trust, London, UK. ${ }^{8}$ Healthplus. ai B.V., Amsterdam, The Netherlands. ${ }^{9}$ Morgan Human Systems, Shrewsbury, UK. ${ }^{10}$ Critical Care Research Group, Nuffield Department of Clinical Neurosciences, University of Oxford, Oxford, UK.

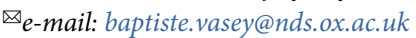

Published online: 1 February 2021

https://doi.org/10.1038/s41591-021-01229-5

\section{References}

1. Keane, P. A. \& Topol, E. J. npj Digital Med. 1, 40 (2018).

2. Collins, G. S. \& Moons, K. G. M. Lancet 393, 1577-1579 (2019).

3. Sounderajah, V. et al. Nat. Med. 26, 807-808 (2020).
4. Liu, X., Rivera, S. C., Moher, D., Calvert, M. J. \& Denniston, A. K. Br. Med. J. 370, m3164 (2020).

5. McCulloch, P. et al. Lancet 374, 1105-1112 (2009).

6. Hirst, A. et al. Ann. Surg. 269, 211-220 (2019).

7. Bilbro, N. A. et al. Ann. Surg. 273, 82-85 (2021).

8. Price, W. N. II, Gerke, S. \& Cohen, I. G. J. Am. Med. Assoc. 322, 1765-1766 (2019).

9. Dalkey, N. \& Helmer, O. Manage, Sci. 9, 458-467 (1963).

10. Powell, C. J. Adv. Nurs. 41, 376-382 (2003).

\section{Competing interests}

X.L. is an industry fellow (observer) with Hardian Health. D.A.C. declares academic grants from GlaxoSmithKline and personal fees from Oxford University Innovation, Biobeats and Sensyne Health, outside the context of this work. B.F.G. is $\mathrm{CEO}$ and Founder of Healthplus.ai and declares consulting fees from NLC Ventures Netherlands. L.M. is director and owner of Morgan Human Systems. P.W. reports grants from National Institute for Health Research, grants from Wellcome, grants from Sensyne Health, and personal fees from Sensyne Health, outside the submitted work.

\title{
Antibiotic resistance: a call to action to prevent the next epidemic of inequality
}

To the Editor - The COVID-19 pandemic has revealed the deadly impacts of structural racism and systemic health inequalities on racial and ethnic minorities in the USA. Black and Hispanic/Latinx populations have been disproportionately impacted by COVID-19, accounting for nearly half of the cases and $37 \%$ of the deaths so far, despite making up less than a third of the US population ${ }^{1}$. This stark imbalance has highlighted the need to examine the role of racial and ethnic disparities in shaping health outcomes.

Antibiotic resistance (AR) is widely considered to be the next global pandemic. When bacteria no longer respond to antibiotics, treatment is more costly and burdensome and is much less likely to succeed. As many as 162,000 US adults die from multidrug-resistant bacterial infections each year, which makes resistant infections the third leading cause of death ${ }^{2}$. Rising concerns about both the health impacts and economic impacts of AR have led to national efforts to increase surveillance, minimize inappropriate antibiotic use, jumpstart the development of diagnostics and antibiotics, and increase awareness of AR. However, the idea that AR could disproportionately impact racial and ethnic minorities has not yet entered the scientific discourse.

The existing literature describing racial and ethnic disparities in antibiotic-resistant infections in the USA is scarce and conflicting. Racial and ethnic data are not routinely collected or checked for accuracy in many clinical settings. Of the few existing studies, some suggest that Black, Hispanic and lower-income people are at higher risk of infection with community-acquired antibiotic-resistant pathogens such as methicillin-resistant Staphylococcus aureus and drug-resistant Streptococcus pneumoniae ${ }^{3,4}$. However, such studies are exceptionally rare. While federal efforts in the past decade have made progress in standardizing the collection and reporting of race and ethnicity data in healthcare settings, many AR-related studies still lack these data.

Nevertheless, there are a number of reasons to suspect that disparities in AR-related morbidity and mortality exist (Fig. 1). For example, while non-Hispanic Black people, Hispanic people and Asian people may receive fewer antibiotic prescriptions over their lifetimes than do non-Hispanic whites ${ }^{5}$, they may also be more likely to consume non-prescription antibiotics ${ }^{6}$. Living in crowded and/ or multigenerational housing, which is more common among racial and ethnic minorities ${ }^{7}$, increases risks of AR acquisition and transmission. Some minority groups may also frequently travel to their native countries, many of which have a high burden of resistant infections ${ }^{8}$. Nearly $60 \%$ of people working in US meat-processing plants are Black or Hispanic/Latinx ${ }^{9}$; occupational contact with 'food animals' may also increase minorities' exposure to zoonotic, resistant pathogens. Finally, with more-frequent underlying comorbidities, racial and ethnic minorities are hospitalized for preventable conditions more often, which puts them at increased risk for drug-resistant hospital-acquired infections. Despite this, the US government's new National Action Plan for Combating Antibiotic-Resistant Bacteria has not prioritized racial or ethnic disparities in AR-related outcomes for either investigation or intervention ${ }^{10}$.

As scientists, researchers and citizens, we have an obligation to ensure that racial and ethnic minorities and economically disadvantaged people will not be disproportionately burdened by the AR crisis. First, we urgently need to understand the scale of underlying disparities in AR-related morbidity and mortality. Continued improvements in the collection of racial and ethnic data in healthcare settings will enable us to evaluate factors underlying disparities across different settings and levels of 'urbanicity'. Second, we must improve AR literacy in low-income and minority communities by incorporating $\mathrm{AR}$ - and infection-prevention education into non-traditional settings. Tailoring future interventions to community settings such as bodegas, tiendas, daycares and classrooms, for example, could help curb unnecessary antibiotic use. Third, we must acknowledge that race or ethnicity is only one factor that might underlie disparities in AR. People who 


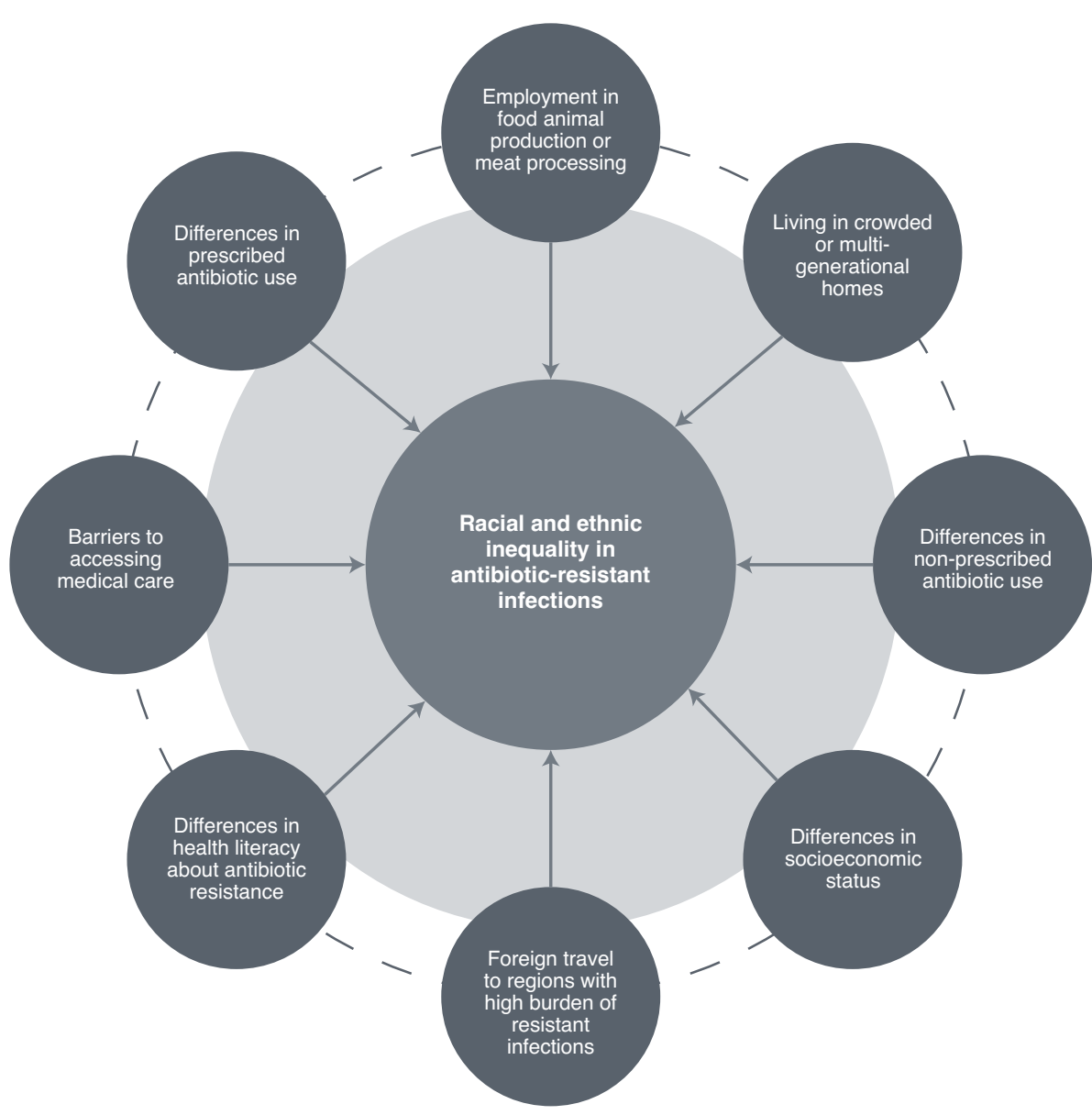

Fig. 1 | Factors that may contribute to racial and ethnic inequality in antibiotic resistance-related morbidity and mortality.

have a low income or are undocumented or uninsured or reside in crowded urban areas may be at risk regardless of their race or ethnicity. Consequently, efforts that seek to improve living conditions, economic stability, education, and access to healthcare in medically underserved areas could drastically slow the AR pandemic. Fourth, we must recognize that disparities in $\mathrm{AR}$ may be driven by global changes. Dietary shifts, increases in urbanization, widening income inequality and global warming could exacerbate disparities across the globe. Finally, we the authors encourage all readers to personally acknowledge and address their own racial and ethnic biases. Given the role of implicit bias on clinical decision-making, it is crucial to critically evaluate and work toward dismantling bias, holding both ourselves and other healthcare professionals accountable for our attitudes and behaviors. A proactive approach that aims to understand, characterize and address the factors that contribute to racial and ethnic inequality in AR has the power not only to inform and guide our response to the next pandemic but also to prevent AR from becoming the next epidemic of inequality.

\section{Maya L. Nadimpalli1,2四, Courtney W. Chan ${ }^{3}$ and Shira Doron ${ }^{2,3}$ \\ ${ }^{1}$ Department of Civil and Environmental Engineering, Tufts University, Medford, MA, USA. ${ }^{2}$ Stuart B. Levy Center for Integrated Management of Antimicrobial Resistance, Tufts University, Boston, MA, USA. ${ }^{3}$ Division of Geographic Medicine and Infectious Diseases, Tufts Medical Center, Tufts University School of Medicine, Boston, MA, USA. $\bigotimes_{e-m a i l: m a y a . n a d i m p a l l i @ t u f t s . e d u}$}

Published online: 18 January 2021 https://doi.org/10.1038/s41591-020-01201-9

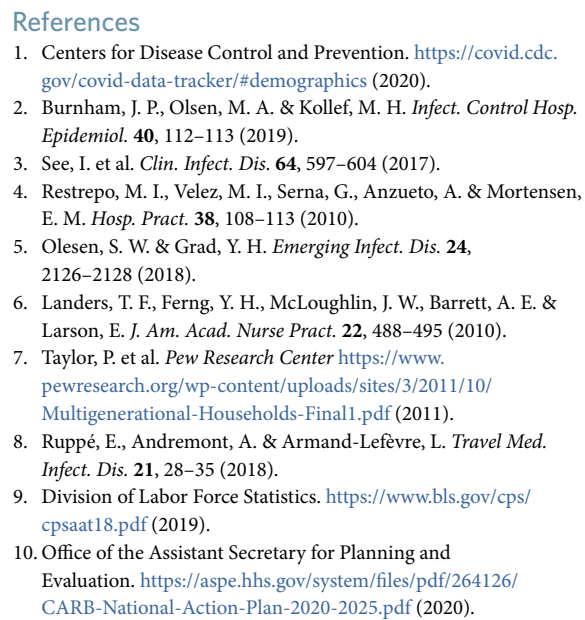

1. Centers for Disease Control and Prevention. https://covid.cdc. gov/covid-data-tracker/\#demographics (2020).

2. Burnham, J. P., Olsen, M. A. \& Kollef, M. H. Infect. Control Hosp. Epidemiol. 40, 112-113 (2019).

3. See, I. et al. Clin. Infect. Dis. 64, 597-604 (2017).

4. Restrepo, M. I., Velez, M. I., Serna, G., Anzueto, A. \& Mortensen, E. M. Hosp. Pract. 38, 108-113 (2010).

5. Olesen, S. W. \& Grad, Y. H. Emerging Infect. Dis. 24 2126-2128 (2018)

6. Landers, T. F., Ferng, Y. H., McLoughlin, J. W., Barrett, A. E. \& Larson, E. J. Am. Acad. Nurse Pract. 22, 488-495 (2010).

7. Taylor, P. et al. Pew Research Center https://www. pewresearch.org/wp-content/uploads/sites/3/2011/10/ Multigenerational-Households-Finall.pdf (2011).

8. Ruppé, E., Andremont, A. \& Armand-Lefèvre, L. Travel Med. Infect. Dis. 21, 28-35 (2018).

9. Division of Labor Force Statistics. https://www.bls.gov/cps/ cpsaat18.pdf (2019)

10. Office of the Assistant Secretary for Planning and Evaluation. https://aspe.hhs.gov/system/files/pdf/264126/ CARB-National-Action-Plan-2020-2025.pdf (2020).

\section{Acknowledgements}

This research was supported in part by the Stuart B. Levy Center for Integrated Management of Antimicrobial Resistance at Tufts (Levy CIMAR), a collaboration of Tufts Medical Center and the Tufts University Office of the Vice Provost for Research (OVPR) Research and Scholarship Strategic Plan (RSSP).

\section{Author contributions}

M.L.N. and S.D. conceived of the idea. All authors developed the first draft, made substantial revisions, and read and approved the final manuscript.

\section{The limits of acceptable political influence over the FDA}

To the Editor - Extensive involvement of the White House in decision-making about the COVID-19 pandemic by the US Food and Drug Administration (FDA) has renewed attention to questions about the agency's independence ${ }^{1}$. Resultant calls to 'follow the science' and avoid 'politicizing' the FDA are rooted in legitimate concerns, but in the context of this pandemic, the relationship among science, values and politics has often been oversimplified. The FDA cannot make decisions on the basis of 